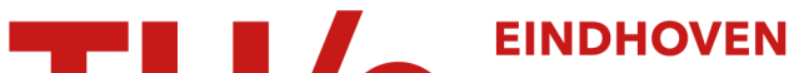 \\ UNIVERSITY OF \\ TECHNOLOGY
}

\section{Approximate self-similarity in models of geological folding}

\section{Citation for published version (APA):}

Budd, C. J., \& Peletier, M. A. (2000). Approximate self-similarity in models of geological folding. SIAM Journal on Applied Mathematics, 60(3), 990-1016. https://doi.org/10.1137/S0036139998337140

DOI:

10.1137/S0036139998337140

Document status and date:

Published: 01/01/2000

\section{Document Version:}

Publisher's PDF, also known as Version of Record (includes final page, issue and volume numbers)

\section{Please check the document version of this publication:}

- A submitted manuscript is the version of the article upon submission and before peer-review. There can be important differences between the submitted version and the official published version of record. People interested in the research are advised to contact the author for the final version of the publication, or visit the $\mathrm{DOI}$ to the publisher's website.

- The final author version and the galley proof are versions of the publication after peer review.

- The final published version features the final layout of the paper including the volume, issue and page numbers.

Link to publication

\section{General rights}

Copyright and moral rights for the publications made accessible in the public portal are retained by the authors and/or other copyright owners and it is a condition of accessing publications that users recognise and abide by the legal requirements associated with these rights.

- Users may download and print one copy of any publication from the public portal for the purpose of private study or research.

- You may not further distribute the material or use it for any profit-making activity or commercial gain

- You may freely distribute the URL identifying the publication in the public portal.

If the publication is distributed under the terms of Article 25fa of the Dutch Copyright Act, indicated by the "Taverne" license above, please follow below link for the End User Agreement:

www.tue.nl/taverne

Take down policy

If you believe that this document breaches copyright please contact us at:

openaccess@tue.nl

providing details and we will investigate your claim. 


\title{
APPROXIMATE SELF-SIMILARITY IN MODELS OF GEOLOGICAL FOLDING*
}

\author{
CHRIS J. BUDD ${ }^{\dagger}$ AND MARK A. PELETIER ${ }^{\ddagger}$
}

Abstract. We propose a model for the folding of rock under the compression of tectonic plates. This models an elastic rock layer imbedded in a viscous foundation by a fourth-order parabolic equation with a nonlinear constraint. The large-time behavior of solutions of this problem is examined and found to be of approximately self-similar form.

Key words. fourth-order parabolic equations, large-time behavior, structural geology, geological folding

AMS subject classifications. 35K55, 35G25, 35B40, 35A05, 58J99, 35Q72, 74Hxx, 74L10, 74K10, 74K20, 74D10

PII. S0036139998337140

1. Introduction. Multilayer geological strata, subjected to the forces imposed on them by the motion of tectonic plates, display a large variety of folding patterns. These include periodic folds, chevron folds, box folds, pinch-and-swell structures, accommodation structures, and many others [11, 18]. Many of these features are understood only at the level of description and a coherent theory describing the mechanisms under which they are produced is still lacking. For the simpler case of single-layer structures in which a thin layer of one type of rock is embedded in a thicker layer of a different rock, the observed features are more homogeneous and the resulting modeling of their formation and evolution is more straightforward. In particular, single-layer folds tend to be localized packets of oscillations with a well-defined (local) wavelength. An example of such is given in Figure 1.1. Localized packets of oscillations have also

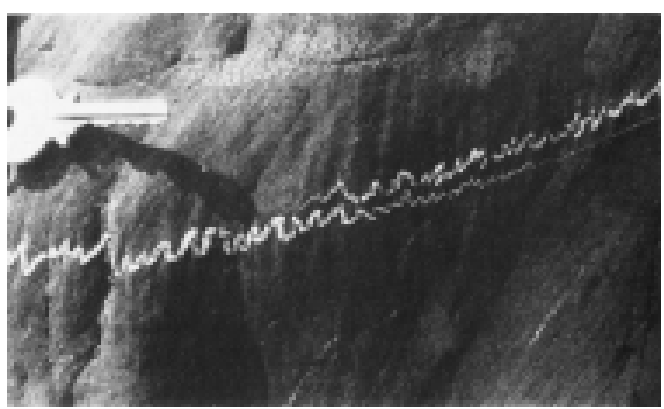

FIG. 1.1. Folded quartz veins in slate. From Porthleven, Cornwall, England (photograph from $[18$, p. 277]).

been found in experimental setups (Figure 1.2). In an important early analysis of the

${ }^{*}$ Received by the editors April 13, 1998; accepted for publication (in revised form) November 5, 1998; published electronically March 10, 2000. This research was supported by EPSRC grant GR/L17177 of the Applied Nonlinear Mathematics Initiative.

http://www.siam.org/journals/siap/60-3/33714.html

${ }^{\dagger}$ Department of Mathematics, University of Bath, Claverton Down, Bath BA2 7AY, UK (c.j.budd@bath.ac.uk).

${ }^{\ddagger}$ Departments of Mathematics and Mechanical Engineering, University of Bath, Claverton Down, Bath BA2 7AY, UK. Current address: CWI, Amsterdam, the Netherlands (Mark.Peletier@cwi.nl). 


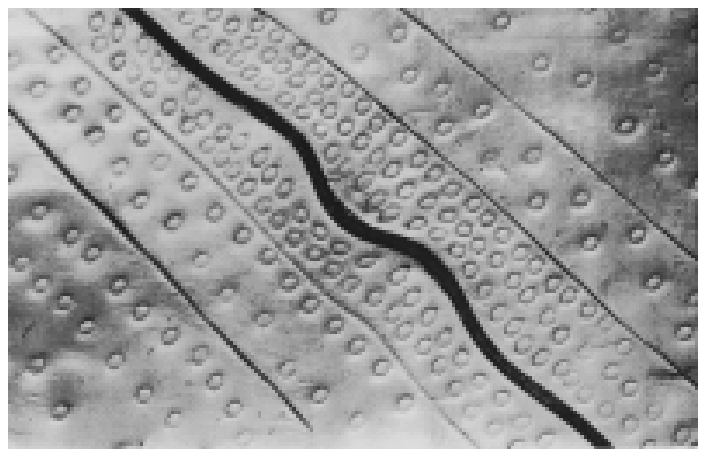

Fig. 1.2. A localized fold in ductile materials (photograph from [19, Plate 13]).

evolution of such patterns, Biot [4] modeled the thin rock layer as a thin (elastic or viscous) strut. Using this approximation and assuming linear constitutive laws and small deflections, Biot predicted the existence of space-periodic folds with a constant (spatial) amplitude. In this analysis the ratio of the wavelength of the folds to the layer thickness depends only on the materials involved and the applied load, and this fact has been widely used to match theory with experiment. However, owing to the lack of reliable data the success of this matching is variable. For an overview of the theory of single-layer folding we refer to $[8,18]$

One of the examples considered in Biot's analysis is that of a single elastic layer of rock embedded in a predominantly viscous material and subject to folding due to tectonic plate movement. This is a common model in the geological literature (see [18, Chapter 11], and the references therein) and the situation that we study in this paper (Figure 1.3). Under a constant force $P$ acting parallel to the layer, Biot showed

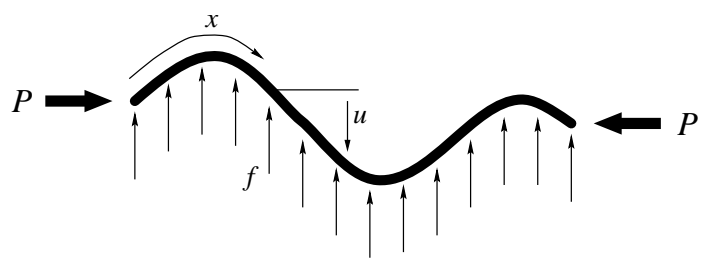

FIG. 1.3. A thin beam under compression.

that sinusoid perturbations of a straight layer grow exponentially in time, with the growth rate being maximal at the so-called dominant wavelength. This wavelength is then assumed to be the one observed in experiments; once a perturbation with a specific wavelength has been singled out, further developments are supposed to continue without changing this wavelength.

There are two shortcomings of this reasoning which we will address in this paper. First, the analysis of Biot does not predict the existence of the localized folding patterns which are often observed in practice. Second, and more fundamentally, Biot's model is not a good representation of the processes which occur when a tectonic plate compresses geological strata, forcing them to fold up. Instead of acting as a constant horizontal force $P$, the effect of the plate motion is to reduce significantly the horizontal space occupied by the strata, in time scales that are comparable to the viscous time scales in the rock. The rock is thus forced to buckle and fold, 
and the force $P$ now becomes a function of time and is implicitly determined by the end-shortening. The evolution of folds under such end-displacement constraints was first investigated in [15].

We model the tectonic compression by supposing that during the motion of the plate, the horizontal space occupied by the rock strata is reduced by an amount $g(t)$, where to a reasonable first approximation

$$
g(t)=a+b t, \quad a \geq 0, \quad b \geq 0 .
$$

Using this approximation we proceed in this paper to study the resulting rock folding patterns and the form of the horizontal load $P(t)$. Throughout our analysis $u=u(x, t)$ is the vertical displacement of the layer and $x$ is the horizontal variable, representing the arc length along the deformed layer, and we presume that deformations are sufficiently small so that

$$
\left|u_{x}\right| \ll 1 \text {. }
$$

Our results differ from those in the existing literature in three significant ways. First, the fold patterns predicted by our analysis are localized and decay for large $x$. Second, the wavelength of the folds increases slowly with time and the patterns evolve in an approximately self-similar manner, showing coherence between spatial and temporal evolutionary structures. Indeed we observe no preferred length scale in our model, leading to similar forms at many scales. Third, the patterns we describe are universal modes of long-term evolution for a wide class of initial data satisfying (1.2).

In this paper we consider two models for the viscous material in which the thin rock layer is embedded. In the first we consider a Winkler model (see Figure 2.1), where the response of the viscous material is considered to be that of a localized viscous dashpot. In the second, we model the effect of a viscous layer acting over a half-space by applying the Hilbert transform to the deformation, thus taking into account the nonlocal response of the viscous layer. The mathematical form of these is given in formulae (2.5) and (2.6). The results and analysis for the two cases are similar.

The variability of the load $P$ leads to an interesting evolution for both types of foundation. At a given load, long waves are amplified at the expense of short ones as a result the stress in the strut and the axial load decrease, shifting the instability to even longer wavelengths. Thus the load decreases to zero while the wavelength and the amplitude increase. In particular, for the Winkler model of a thin elastic layer lying between two viscous layers and subject to end-shortening, we prove the following result about the deflection $u(x, t)$ and horizontal load $P(t)$.

TheOREM 1.1. For large times,

$$
P(t) \sim A \sqrt{\frac{\log (t)}{t}}
$$

and

$$
u(x, t) \sim B P(t)^{-1 / 4} g(t)^{1 / 2} \log (t)^{-1 / 4} e^{-\alpha y^{2} / \log (t)} \cos (y),
$$

where

$$
y=x P(t)^{1 / 2}
$$


and the values of $A, B$, and $\alpha$ depend upon $a$ and $b$ in (1.1). The spatial derivative $u_{x}$ has the long-term behavior

$$
u_{x}(x, t) \sim-B P(t)^{1 / 4} g(t)^{1 / 2} \log (t)^{-1 / 4} e^{-\alpha y^{2} / \log (t)} \sin (y) .
$$

See Theorems 5.1 and 4.2 for exact statements.

Roughly speaking, the wavelength of the folds is proportional to $t^{1 / 4}$ with a logarithmic correction, and $P$ is proportional to $t^{-1 / 2}$ again with a logarithmic correction. A typical such pattern of folding is given in Figure 1.4. Observe from Theorem 1.1

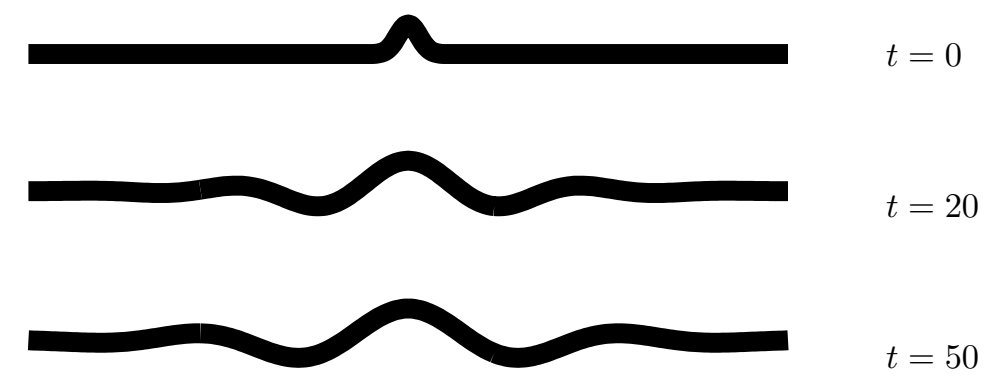

FIG. 1.4. Evolution of a deformed layer (Winkler model). Constant end-shortening, initial data was $e^{-x^{2}}$. Compare this figure to Figure 1.2.

that the maximum of the derivative $u_{x}$ is roughly proportional to $t^{-1 / 8} g(t)^{1 / 2}$. If $b=0$, then this derivative tends to zero, implying that the long-term limit is consistent with the approximation (1.2). However, if $b \neq 0$, it represents an intermediate asymptotic description and breaks down for very large times, at which point geometric nonlinearities start to dominate the behavior. The effects of these form the basis of a subsequent paper.

The layout of the remainder of this paper is as follows. In section 2 we introduce the basic model for the folding of an elastic rock layer under prescribed end-shortening in both a viscous Winkler and a viscous half-space formulation. In section 3 we show that both models are well posed and give smooth solutions which exist for all time. In sections 4 and 5 we consider the long-time behavior of, respectively, $P(t)$ and $u(x, t)$ in the Winkler model and derive the results that are stated in Theorem 1.1. In section 6 we repeat our calculations for the half-space model. In section 7 we account for the existence of the logarithmic terms in (1.3), (1.4) by showing that true selfsimilar solutions of the model equations do not exist. Finally in section 8 we describe a finite-element numerical method for solving the model problem and compare our numerical calculations with the asymptotic predictions.

2. The model. Natural rock has a complex response to deformation. The shorttime, small-strain response has been extensively studied in laboratory setups and is elastic in nature. Seismic waves are an illustration of this property. On longer time scales, and under larger strains, permanent deformation is observed, but without being easily classified as a simple elastoplastic or viscoelastic behavior. The picture is complicated by the large variety in materials and structural makeup, giving rise to a subtle interplay between microscopic and macroscopic qualities. To complete the difficulties, most of the folding is spread out over long times and occurs under 
high pressure and temperature; material properties depend strongly on these two parameters, experimental determination under those conditions is difficult, and the actual values are usually unknown.

When facing these difficulties, a common choice is to treat rocks as viscoelastic materials. The time scales involved vary from hundreds to millions of years. Although naturally reliable data are difficult to obtain, it seems reasonable that both elastic and viscous effects contribute to the structures that we observe (cf. [18, p. 302]). In a composite structure consisting of a thin layer embedded in a much thicker layer (called foundation, bedding, or matrix) we expect that the viscosities of the two materials involved will be different, and that there will be time scales on which we see elastic behavior in one material and viscous behavior in the other. It is this situation that we want to investigate here, and we therefore make the simplifying assumption that the matrix is viscous and the layer is elastic.

One might object to this model that in the deformations depicted in Figures 1.1 and 1.2 the material is deformed past the plastic limit and therefore does not behave in an elastic fashion. This is certainly true; however, there are reasons why the model of an elastic layer in a viscous medium is relevant nonetheless. First, it is a classical model in structural geology; there is a considerable body of theoretical work based on this configuration (e.g., [3, 4, 18, 14, 15]), in which, however, the load is invariably fixed (with the notable exception of [15]). The juxtaposition of the results for the fixed end-displacement model considered in this paper gives an interesting insight in the influence of the boundary conditions on the behavior of the model. Second, it is often argued that wavelength selection happens in the first increments of folding (this is the basis for the "dominant wavelength" school of theory). During these initial stages the deformation can reasonably be supposed to be elastic. Despite the subsequent plastic amplification, the initial elastic stages therefore have a significant influence on the final folds. A similar feature is observed in other materials and other geometries. A notable example is the axially compressed cylinder $[12,13,9]$, where the geometry of the final, plastic deformation pattern is also determined by the elastic initial stages of deformation.

We envisage the following model situation. A thin elastic layer of constant thickness lies embedded in a viscous medium, with a no-slip condition at the boundary. The rest position of the layer is a straight line, but at $t=0$ the layer deviates slightly from this position, and consequently it exerts a force on the viscous medium. As the system evolves, this force causes the viscous bedding to move, and by the no-slip condition the layer moves with it.

The instantaneous initial perturbation is motivated by the elastic short-term response of natural rock. For a straight layer under parallel compression, folding typically starts with an elastic buckle which subsequently evolves slowly in a viscous manner $[10,22,23]$. Since the time scale in our modeling is that of this viscous response, the initial buckle is effectively an instantaneous event. We intend to make a detailed study of the initial stages of the evolution in a fully viscoelastic context in a future paper.

We model the elastic layer as a thin inextensible elastic beam that buckles under the layer-parallel force $P$ and undergoes lateral forcing $f$ (from the viscous foundation), as in Figure 1.3. We define $u$, the vertical displacement, as a function of $x$, the curvilinear coordinate measuring arc length along the layer, and assume that the lateral force $f$ is independent of $u$ ( $f$ will in fact depend on $u_{t}$, since the matrix is viscous). 
Following [20, p. 27], the total potential energy $W$ of a given configuration is equal to

$$
W=U_{s}+U_{f}-P S,
$$

where $U_{s}$ is the strain energy of the deformed beam, $U_{f}$ is the potential energy associated with the force field $f$, and $S$ is the end-shortening of the beam (the difference between original length $2 L$ and the horizontal extent after deformation). Here the bending strain energy is given by

$$
U_{s}=\frac{1}{2} E I \int_{-L}^{L} \chi(x)^{2} d x
$$

where $E I$ is the bending stiffness and $\chi$ is the curvature, given by

$$
\chi=\frac{u_{x x}}{\sqrt{1-u_{x}^{2}}}
$$

and $2 L$ is the total length of the beam. Throughout this article we use subscripts to denote differentiation. The foundation energy is

$$
U_{f}=\int_{-L}^{L} f(x) u(x) d x
$$

and the end-shortening of the beam is given by (note that $x$ denotes arc length)

$$
\begin{aligned}
S & =L-\int_{-L}^{L} \sqrt{1-u_{x}^{2}} d x \\
& =\int_{-L}^{L}\left\{1-\sqrt{1-u_{x}^{2}}\right\} d x .
\end{aligned}
$$

Thus the total potential energy is

$$
W=\int_{-L}^{L}\left[\frac{1}{2} E I \frac{u_{x x}^{2}}{1-u_{x}^{2}}-P\left\{1-\sqrt{1-u_{x}^{2}}\right\}+f u\right] d x .
$$

This derivation assumes that the layer is long in comparison to its thickness. We shall now add an additional assumption: that the layer is also long in comparison to the displacement, i.e., that deformation is confined to a small part of the layer. It is then reasonable to consider layers of infinite length, by setting $L=\infty$ in the integral above. In sections 4 and 5 we will see that solutions for the infinite layer spread out indefinitely as time tends to infinity. Obviously, this will not be the case for a bounded layer, but we expect that this spreading behavior will become apparent between the initial phase and the moment when the boundary conditions begin to be felt. Barenblatt [1] has termed this intermediate asymptotics. In order to characterize the long-term behavior, we will from now on suppose that the layer has infinite length and that all integrals are on $\mathbb{R}$.

At this stage in the modeling we also make the assumption that $u_{x}$ is small. This allows us to replace

$$
\frac{u_{x x}^{2}}{1-u_{x}^{2}} \quad \text { by } \quad u_{x x}^{2}
$$


and

$$
1-\sqrt{1-u_{x}^{2}} \quad \text { by } \quad \frac{1}{2} u_{x}^{2}
$$

so that we obtain the "linearized" potential energy

$$
\mathcal{W}=\int_{-\infty}^{\infty}\left[\frac{1}{2} E I u_{x x}^{2}-\frac{1}{2} P u_{x}^{2}+f u\right] d x
$$

The equations of motion are derived by seeking a stationary point of this energy functional. This leads directly to the differential equation

$$
E I u_{x x x x}+P u_{x x}+f=0, \quad-\infty<x<\infty .
$$

We envisage coming back to the geometrically nonlinear model (2.1) in a later publication.

We now come to the viscous matrix, which defines the function $f$. We consider two different models: a Winkler foundation and a viscous half-space foundation (see Figure 2.1). The Winkler foundation consists of a continuous array of viscous dash-

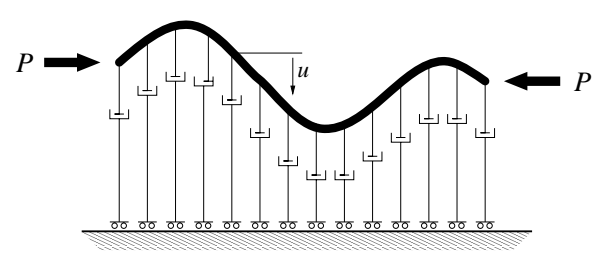

(a) Winkler foundation

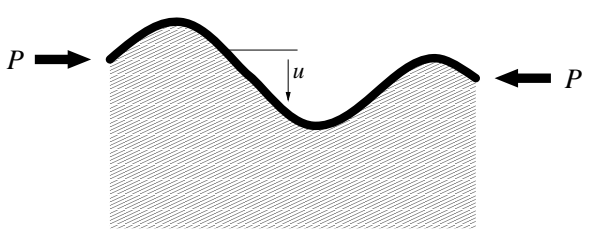

(b) Halfspace foundation

FIG. 2.1. Foundation types.

pots, which are free to move horizontally but are constrained vertically. The half-space is simply a compressible viscous fluid that fills the half-space delimited by the beam. In geological structures these foundations are, of course, on both sides of the beam; but the functions $f$ that we derive below are independent of the side of the beam that the foundation is on, and we can therefore combine the two foundations into one without any loss of generality.

If the dashpots in the Winkler foundation have a viscosity $\eta$, then the bedding force $f$ is linked to the displacement $u$ by

$$
f=\eta u_{t}
$$

For the viscous half-space the relation is no longer local. Biot [4] derives the following 
relation in terms of the Fourier transforms ${ }^{1} \hat{f}$ and $\hat{u}_{t}$ :

$$
\hat{f}(\omega)=4 \eta|\omega| \hat{u}_{t}, \quad \omega \in \mathbb{R} .
$$

Here $\eta$ is the viscosity of the material. Using the Hilbert transform $H$, this can be formulated in the spatial domain as

$$
f=4 \eta H\left(u_{x t}\right) .
$$

We can choose either (2.3) or (2.4) to close the system. In both cases, if we specify $P$, the axial load, and an initial configuration $u_{0}$, then the combination of $(2.2)$ and the foundation relation $((2.3)$ or $(2.4))$ forms a well-posed problem. This is the model studied extensively in the literature, as mentioned in the introduction.

In this paper we wish to investigate a different problem. As mentioned above, tectonic compression is assumed to be the driving force of the folding. It therefore seems more natural to model the folding as a prescribed end-shortening process rather than as a prescribed- $P$ process. If we prescribe the end-shortening of the layer, then the load $P$ needs to be determined as part of the solution.

Using the linearized form of the end-shortening $S$,

$$
\mathcal{S}=\frac{1}{2} \int_{-\infty}^{\infty} u_{x}^{2} d x
$$

we can formulate this model as a system of equations in the unknowns $u$ and $P$. First the Winkler foundation: we find

$$
\begin{aligned}
& \eta u_{t}+E I u_{x x x x}+P u_{x x}=0, \quad-\infty<x<\infty, \quad t>0, \\
& \frac{1}{2} \int_{-\infty}^{\infty} u_{x}^{2} d x=g(t),
\end{aligned}
$$

where $g(t)$ is the prescribed end-shortening. With the tectonic origin in mind, a typical function $g$ might be $g(t)=a+b t(a, b \geq 0)$. For the half-space foundation the model becomes

$$
\begin{aligned}
& 4 \eta H\left(u_{x t}\right)+E I u_{x x x x}+P u_{x x}=0, \quad-\infty<x<\infty, \quad t>0 \\
& \frac{1}{2} \int_{-\infty}^{\infty} u_{x}^{2} d x=g(t) .
\end{aligned}
$$

At this point we might make a remark about the time scales involved. As an example of a typical fold we choose characteristic scales $h=10 \mathrm{~cm}$ (the thickness of the layer) and $x^{*}=1 \mathrm{~m}$ (the characteristic horizontal length). $I=h^{3} /(12(1-$ $\left.\nu^{2}\right)$ ), and common values for the elastic parameters of rocks are $E=10^{10} \mathrm{~Pa}$ and $\nu=0.2$. The viscosity poses a real challenge: estimates vary from $10^{13} \mathrm{Pas}[5$, Vol. 2,

\footnotetext{
${ }^{1}$ We define the Fourier transforms on $L^{2}(\mathbb{R})$ as follows:

$$
\hat{u}(\omega)=\frac{1}{\sqrt{2 \pi}} \int_{\mathbb{R}} u(x) e^{-i \omega x} d x \quad \text { and } \quad(\hat{u})(x)=\frac{1}{\sqrt{2 \pi}} \int_{\mathbb{R}} \hat{u}(\omega) e^{i \omega x} d \omega .
$$
}

The Hilbert transform $H: L^{2}(\mathbb{R}) \rightarrow L^{2}(\mathbb{R})$ is given by either of the formulas

$$
H(u)(x)=\frac{1}{\pi} \int_{\mathbb{R}} \frac{u(y)}{x-y} d y \quad \text { or } \quad(H(u))^{\gamma}(\omega)=-i \operatorname{sgn}(\omega) \hat{u}(\omega) .
$$

The integral is to be interpreted in the sense of the principal value. 
Table 2, p. 174] via $10^{20} \mathrm{Pas}\left[11\right.$, p. 28] to $10^{22} \mathrm{Pas}[18$, p. 301]. Here we take the average of the two extremes, $3 \cdot 10^{17} \mathrm{Pas}$. Setting $x=x^{*} \bar{x}$ and $t=t^{*} \bar{t}$ we find the equation

$$
u_{\overline{x x x x}}+\frac{9 x^{*}}{E h^{3}} P u_{\overline{x x}}+\frac{46.08 \eta\left(x^{*}\right)^{3}}{t^{*} E h^{3}} H\left(u_{\bar{x} \bar{t}}\right)=0 .
$$

The corresponding characteristic time scale $t^{*}$ is given by

$$
t^{*}=\frac{46.08 \eta\left(x^{*}\right)^{3}}{E h^{3}} \approx 46,000 \text { years. }
$$

Geological time scales tend to be much longer than this, and as a consequence we expect that observed features reflect the long-term behavior in this model.

3. Well posedness. This section and the following are devoted to the well posedness and long-term behavior of the Winkler foundation model,

Find $(u, P)=(u(x, t), P(t))$ such that

$$
u_{t}+u_{x x x x}+P u_{x x}=0, \quad x \in \mathbb{R}, t>0
$$

with initial condition

$$
u(x, 0)=u_{0}(x), \quad x \in \mathbb{R}
$$

such that $u$ satisfies the constraint

$$
\frac{1}{2} \int_{\mathbb{R}} u_{x}^{2} d x=g(t), \quad t>0 .
$$

For the formulation of the theorem below, the Sobolev spaces $H^{k}(\mathbb{R})$ are convenient. The space $H^{k}(\mathbb{R}), k \geq 0$, is defined as the set of functions $f \in L^{2}(\mathbb{R})$ such that the $k$ th derivative of $f$ is in $L^{2}(\mathbb{R})$, or equivalently, such that the integral

$$
\int_{\mathbb{R}}\left(1+\omega^{2}\right)^{k}|\hat{f}(\omega)|^{2} d \omega
$$

is finite.

THEOREM 3.1. Let $u_{0} \in H^{1}(\mathbb{R})$. Suppose that $g \in C([0, T)) \cap C^{n+1}([0, T))$ $(n \geq 0,0<T \leq \infty)$ satisfies $g>0$ and $g(0)=\frac{1}{2} \int u_{0 x}^{2}$. Then there exists a unique solution $(u, P)$ such that

(1) $u \in C\left([0, T) ; H^{1}(\mathbb{R})\right) \cap C^{n+1}\left((0, T) ; H^{k}(\mathbb{R})\right)$;

(2) $P \in L^{1}(0, T) \cap C^{n}((0, T))$.

Proof. We formulate the problem as an integral equation for $\Pi(t)=\int_{0}^{t} P(s) d s$. Applying the Fourier transform (in space) to (3.1), we find that the transformed unknown $\hat{u}$ satisfies the ordinary differential equation

$$
\hat{u}_{t}+\left(\omega^{4}-P(t) \omega^{2}\right) \hat{u}=0
$$

which, for given $P$, has the solution

$$
\hat{u}(\omega, t)=\hat{u}_{0}(\omega) e^{-\omega^{4} t+\Pi(t) \omega^{2}} .
$$


In terms of $\hat{u}$, the constraint can then be written

$$
\frac{1}{2} \int \omega^{2} e^{-2 \omega^{4} t+2 \Pi(t) \omega^{2}}\left|\hat{u}_{0}(\omega)\right|^{2} d \omega=g(t) .
$$

Finding a solution $(u, P)$ to the original problem now corresponds to finding a function $\Pi(t)$ that satisfies this integral equation. Note that the two formulations are only equivalent if $\Pi$ has sufficient regularity for differentiation.

The function

$$
(t, y) \mapsto G(t, y) \stackrel{\text { def }}{=} \frac{1}{2} \int \omega^{2} e^{-2 \omega^{4} t+2 y \omega^{2}}\left|\hat{u}_{0}(\omega)\right|^{2} d \omega
$$

is strictly increasing in $y$, and for $t>0$ fixed, $G(t, y) \rightarrow \infty$ as $y \rightarrow \infty$ and $G(t, y) \rightarrow 0$ as $y \rightarrow-\infty$. Hence there exists for every $t>0$ exactly one $\Pi(t) \in \mathbb{R}$ such that $G(t, \Pi(t))=g(t)$. Note that this proves the uniqueness of $\Pi$ and therefore of the function $P$. Also remark that as $t \rightarrow 0, \Pi(t) \rightarrow 0$.

For the regularity of the function $\Pi$ we employ the implicit function theorem. Since both partial derivatives

$$
\begin{aligned}
& \frac{\partial G}{\partial y}=\int \omega^{4} e^{-2 \omega^{4} t+2 y \omega^{2}}\left|\hat{u}_{0}(\omega)\right|^{2} d \omega, \\
& \frac{\partial G}{\partial t}=-\int \omega^{6} e^{-2 \omega^{4} t+2 y \omega^{2}}\left|\hat{u}_{0}(\omega)\right|^{2} d \omega
\end{aligned}
$$

are defined, bounded, and bounded away from zero whenever $t>0$, the function $\Pi$ has the same degree of regularity as $g$. Consequently, $P=\Pi^{\prime} \in C^{n}((0, T))$.

The function $u$ mentioned in the theorem is obtained by inverting the Fourier transform in (3.4). The regularity of $u$ follows from the strong decay of $\omega^{2} e^{-2 \omega^{4} t+2 y \omega^{2}}$ as $|\omega| \rightarrow \infty$. The continuity of $u$ at $t=0$ follows from $\lim _{t \rightarrow 0} \Pi(t)=0$.

Remark 3.1. It follows from the application of the implicit function theorem that if $g$ is analytic, then $P$ is also an analytic function of $t$.

Remark 3.2. If $u_{0} \in H^{3}(\mathbb{R})$, then the partial derivatives of $G$ are both finite and nonzero at $(t, y)=(0,0)$, implying that the function $\Pi$ is continuously differentiable up to $t=0$. Consequently, the axial load $P=\Pi^{\prime}$ is continuous up to $t=0$. If $u_{0} \notin H^{3}(\mathbb{R})$, then it may happen that $P$ is unbounded at $t=0$. This is also apparent from part (1) of the theorem below.

THEOREM 3.2. Let $(u, P)$ be the solution given by Theorem 3.1. The following statements hold, provided that $g$ has the appropriate regularity:

(1) $P=\left[g^{\prime}(t)+\int u_{x x x}^{2}\right]\left(\int u_{x x}^{2}\right)^{-1}$;

(2) $P^{\prime}=\left[g^{\prime \prime}(t)-2 \int u_{x t}^{2}\right]\left(\int u_{x x}^{2}\right)^{-1}$.

Proof. Part (3.2) follows from differentiating the constraint

$$
g^{\prime}(t)=\frac{1}{2} \frac{d}{d t} \int u_{x}^{2}=-\int u_{x x x}^{2}+P(t) \int u_{x x}^{2}
$$

By differentiating this expression again we find

$$
\begin{aligned}
g^{\prime \prime}(t) & =P^{\prime} \int u_{x x}^{2}+2 P \int u_{x x} u_{x x t}-2 \int u_{x x x} u_{x x x t} \\
& =P^{\prime} \int u_{x x}^{2}+2 \int u_{x t}^{2}
\end{aligned}
$$


which proves part (2).

Remark 3.3. A different way of viewing the well posedness of this system is as an example of constrained gradient flow. Recalling the definition of the "linearized" elastic strain energy

$$
\mathcal{U}_{s}=\frac{1}{2} \int u_{x x}^{2}
$$

and end-shortening

$$
\mathcal{S}=\frac{1}{2} \int u_{x}^{2}
$$

we can view system (3.1)-(3.2) as a flow following the gradient of $\mathcal{U}_{s}$ subject to the constraint $\mathcal{S}=g(t)$. Such a flow satisfies an equation of the form

$$
u_{t}=-\nabla \mathcal{U}_{s}(u)+P \nabla \mathcal{S}(u),
$$

where $P$ is a Lagrange multiplier. Interpreting - formally- $\nabla \mathcal{U}_{s}(u)$ as $u_{x x x x}$ and $\nabla \mathcal{S}(u)$ as $-u_{x x}$, we find this equation to be identical to (3.1).

To make an initial estimate of the long-term behavior we observe that (3.1) is invariant under the scaling

$$
t \rightarrow \lambda t, \quad x \rightarrow \lambda^{1 / 4} x, \quad P \rightarrow \lambda^{-1 / 2} P .
$$

This invariance suggests a self-similar structure of the form

$$
u(x, t) \sim t^{\alpha} f\left(x t^{-1 / 4}\right), \quad P \sim Q t^{-1 / 2} .
$$

The value of $\alpha$ results from the constraint: if $g(t)$ behaves like $t^{\gamma}$ for large $t$, then from (3.2) we find that $\alpha$ should be equal to $1 / 8+\gamma / 2$,

$$
\int u_{x}^{2} d x \sim t^{1 / 4+\gamma} \int t^{-1 / 2} f^{\prime}\left(x t^{-1 / 4}\right)^{2} d x \sim t^{\gamma} \int f^{\prime}(\eta)^{2} d \eta .
$$

As an example, in the case of constant $g$ one might expect the long-term behavior of (3.1)-(3.2) to be given by a self-similar solution that follows this scaling, i.e., $\alpha=1 / 8$,

$$
u(x, t) \sim t^{1 / 8} f\left(x t^{-1 / 4}\right), \quad P \sim Q t^{-1 / 2} .
$$

The numerical evidence points in the same direction (Figure 3.1).

However plausible this may seem, it is not true; there exists no self-similar solution of this kind, as we show in section 7 . Although as a consequence the long-term behavior is not self-similar, we show in the next two sections that - just like the numerical results suggest - the self-similar scaling does provide a good first approximation. The situation is reminiscent of that in parabolic blow-up problems, where in a comparable way the blow-up profile is approximately self-similar [7, 2].

4. Long-term behavior: The load $\boldsymbol{P}$. Throughout this and the following sections we shall use the notation

$$
f(t) \sim g(t) \quad \text { as } t \rightarrow \infty
$$



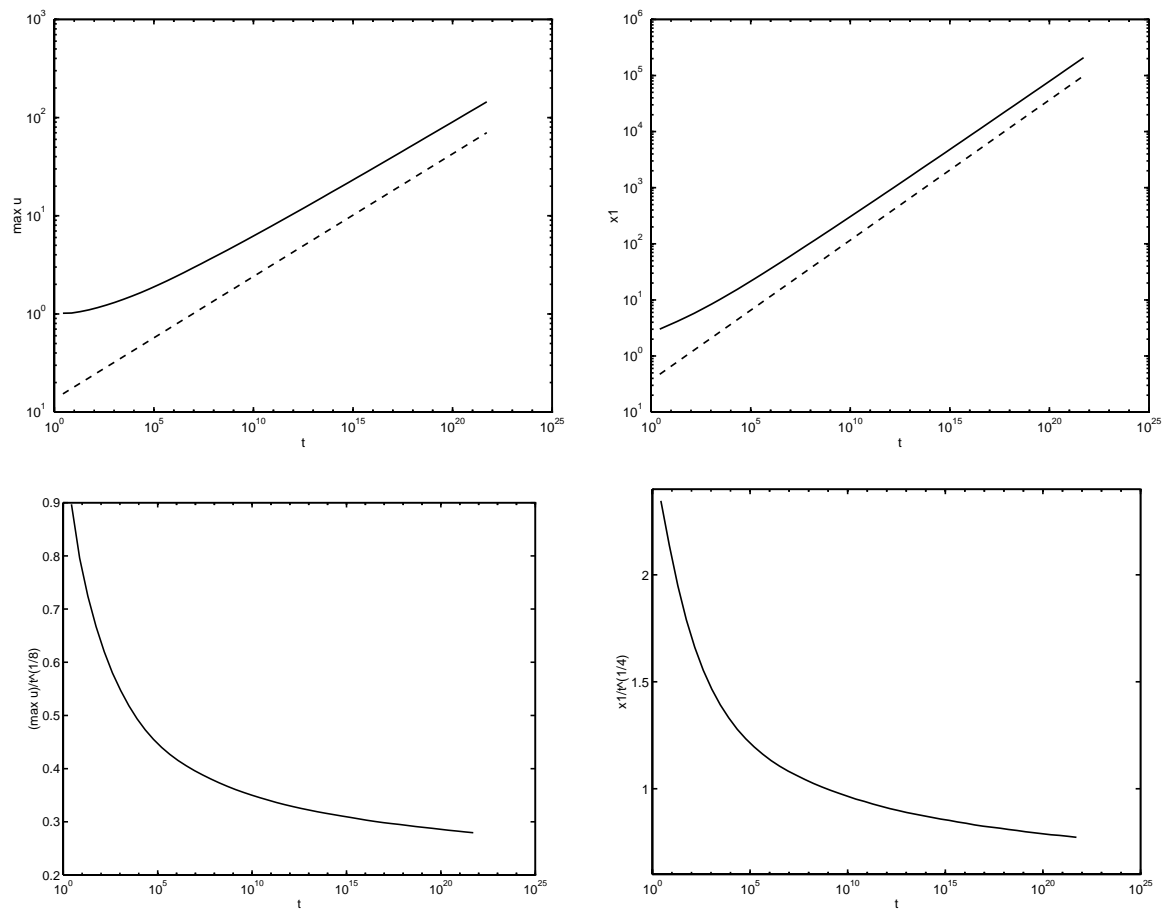

FIG. 3.1. To first order, the numerical results suggest self-similar long-term behavior (top row). On the left is plotted the maximal value of $u$ against $t$, on the right the location of the first zero $x_{1}$ of the solution. The dashed lines correspond to $t^{1 / 8}$ and $t^{1 / 4}$. On the bottom row it can be seen that after appropriate rescaling a slow drift is noticeable, showing that the long-term behavior is only approximately self-similar. (Constant end-shortening, initial data $e^{-x^{2}}$; see section 8 for the numerical methods used.)

to denote that the quotient $f(t) / g(t)$ tends to unity in the limit $t \rightarrow \infty$; the notation

$$
f(t)=o(g(t)) \quad \text { as } t \rightarrow \infty
$$

means that the quotient tends to zero.

Equation (3.5) provides a convenient way of investigating the large-time behavior of solutions. By estimating the behavior of the integral as a function of $\Pi$, for large $\Pi$, we can express the long-term behavior of $\Pi$ in the long-term behavior of $g$. This is, in summary, the content of this section.

We shall use "Laplace's method," also called Watson's lemma, to calculate the asymptotic behavior of integrals. Loosely formulated, this method states that if $p(\omega)$ takes its minimum at $\omega=a$, with $p^{\prime \prime}(a)>0$ and $q(a) \neq 0$, then

$$
\int_{I} q(\omega) e^{-\tau p(\omega)} d \omega \sim \sqrt{2 \pi} q(a) \frac{e^{-\tau p(a)}}{\sqrt{p^{\prime \prime}(a) \tau}} \quad \text { as } \tau \rightarrow \infty,
$$

where $I$ can be any open interval containing $a$. A precise formulation of this result, along with its proof, can be found in [16, Theorem 7.1].

In the course of the proof of the next theorem we shall use the functions

$$
F_{n}(y) \stackrel{\text { def }}{=} \int \xi^{2 n+2} e^{-2 \xi^{4}+2 y \xi^{2}} d \xi,
$$


defined for $n \geq 0$ and $y \in \mathbb{R}$. We summarize a number of useful properties in the following lemma.

LEMMA 4.1.

(1) $F_{n}(y) \sim 2^{-n-1} \sqrt{\pi} y^{n+1 / 2} e^{y^{2} / 2}$ as $y \rightarrow \infty$;

(2) $F_{n}(y) \sim 2^{-n} y^{n} F_{0}(y)$ as $y \rightarrow \infty$;

(3) $F_{n}^{-1}(z) \sim \sqrt{2}(\log z)^{1 / 2}$ as $z \rightarrow \infty$.

Proof. Part (1) follows from applying (4.1) to a transformed version of $F_{n}$,

$$
F_{n}(y)=y^{n+3 / 2} \int \eta^{2 n+2} e^{-2 y^{2}\left(\eta^{4}-\eta^{2}\right)} d \eta .
$$

Part (2) is obtained by applying part (1) twice. Part (3) is a direct consequence of part (1).

THEOREM 4.2. Let $u_{0} \in H^{1}(\mathbb{R})$ be such that $\hat{u}_{0}(\omega)$ is bounded for $\omega \in \mathbb{R}$ and continuous at $\omega=0$, and that $\hat{u}_{0}(0) \neq 0$. In addition, assume that $g(t) \sim C t^{\gamma}$ for some $C>0$ and $\gamma>-3 / 4$, and that $g^{\prime}(t)$ is of at most polynomial growth. Then

$$
P(t) \sim \frac{1}{2} t^{-1 / 2} \sqrt{2(\gamma+3 / 4) \log t} \quad \text { as } t \rightarrow \infty .
$$

Remark 4.1. The condition on the regularity of the Fourier transform of the initial datum translates into the spatial domain as a condition on the decay of the tails at plus and minus infinity; for instance, if $u_{0} \in L^{1}(\mathbb{R})$, then $\hat{u}_{0}$ is continuous on $\mathbb{R}$.

Remark 4.2. The condition $\gamma>-3 / 4$ is critical for the behavior of the function $h(t)$ defined below. For $\gamma>-3 / 4, h(t)$ tends to infinity as $t \rightarrow \infty$, while for $\gamma \leq$ $-3 / 4$ the function $h(t)$ remains bounded. This difference has important consequences for the subsequent analysis. In addition, there is a possibility for exact self-similar behavior when $\gamma \leq-3 / 4$ (see the remark at the end of section 7). From the point of view of interpretation, $\gamma>0$ implies shortening of the strut as time evolves, while $\gamma<0$ implies a lengthening; the critical value $\gamma=-3 / 4$ seems to have no specific interpretation.

Proof. First we prove that $\Pi(t) / t \rightarrow 0$ as $t \rightarrow \infty$. Suppose that this is not the case; we can then find a sequence $t_{n} \rightarrow \infty$ and $c>0$ such that $\Pi\left(t_{n}\right) \geq c t_{n}$ for all $t_{n}$. Using the continuity of $\hat{u}_{0}$ at zero we choose $\varepsilon>0$ such that

$$
\left|\hat{u}_{0}(\omega)\right|^{2} \geq \frac{1}{2} \hat{u}_{0}^{2}(0) \quad \text { for } \quad-\varepsilon<\omega<\varepsilon .
$$

Note that since $u_{0}$ is real, $\hat{u}_{0}(0)=\int u_{0}$ is also real. Then, recalling (3.5),

$$
\begin{aligned}
g(t) & =\frac{1}{2} \int \omega^{2} e^{-2 \omega^{4} t_{n}+2 \Pi\left(t_{n}\right) \omega^{2}}\left|\hat{u}_{0}(\omega)\right|^{2} d \omega \\
& \geq \frac{1}{4} \hat{u}_{0}^{2}(0) \int_{-\varepsilon}^{\varepsilon} \omega^{2} e^{\left(-2 \omega^{4}+2 c \omega^{2}\right) t_{n}} d \omega,
\end{aligned}
$$

and this expression tends to infinity exponentially as $t_{n} \rightarrow \infty$, implying a contradiction with the polynomial growth of $g$. Therefore, $\Pi(t) / t \rightarrow 0$ as $t \rightarrow \infty$.

By introducing the change of variables $\omega=\xi t^{-1 / 4}, \Pi(t)=t^{1 / 2} \Sigma(t)$, (3.5) can be reformulated as

$$
\frac{1}{2} \int \xi^{2} e^{-2 \xi^{4}+2 \Sigma(t) \xi^{2}}\left|\hat{u}_{0}\left(\xi t^{-1 / 4}\right)\right|^{2} d \xi=t^{3 / 4} g(t) \stackrel{\text { def }}{=} h(t) .
$$


We first determine the large-time behavior of $\Sigma$. Since for large $t$ the value of $\hat{u}_{0}\left(\xi t^{-1 / 4}\right)$ approaches $\hat{u}_{0}(0)$ on bounded sets, we wish to approximate the first by the second,

$$
\int \xi^{2} e^{-2 \xi^{4}+2 \Sigma(t) \xi^{2}}\left|\hat{u}_{0}\left(\xi t^{-1 / 4}\right)\right|^{2} d \xi \sim \hat{u}_{0}^{2}(0) F_{0}(\Sigma(t)) .
$$

We use the following technical lemma to make this precise.

Lemma 4.3. If $v \in L^{\infty}(\mathbb{R})$ is continuous at zero, $v(0) \neq 0$, then in the limit $y, p \rightarrow \infty, y=o\left(p^{2}\right)$, we have

$$
\int \xi^{2 n+2} e^{-2 \xi^{4}+2 y \xi^{2}} v(\xi / p) d \xi \sim v(0) F_{n}(y)
$$

We postpone the proof of this lemma until later in this section.

To apply this lemma to (4.3) we need to show that $\Sigma(t)=o\left(t^{1 / 2}\right)$ as $t \rightarrow \infty$. We showed above that the quotient $\Pi(t) / t$ tends to zero in the limit $t \rightarrow \infty$; consequently, the same holds for $\Sigma(t) t^{-1 / 2}=\Pi(t) / t$, and therefore $y=\Sigma$ and $p=t^{1 / 4}$ fulfill the requirements of the lemma. Consequently,

$$
F_{0}(\Sigma(t)) \sim \hat{2} u_{0}^{-2}(0) h(t) .
$$

Inverting $F_{0}$ (see Lemma 4.1), we find that

$$
\Sigma(t) \sim \sqrt{2 \log h(t)} \sim \sqrt{2(\gamma+3 / 4) \log t} \quad \text { as } t \rightarrow \infty .
$$

Note that this expression is completely independent of $u_{0}$. We will discuss the influence of the initial data on the asymptotic behavior in more detail later on.

Next we determine the large-time behavior of $P$. Using part (3.2) of Theorem 3.2,

$$
P(t) \int \omega^{4} e^{-2 \omega^{4} t+2 \Pi(t) \omega^{2}}\left|\hat{u}_{0}(\omega)\right|^{2} d \omega=\int \omega^{6} e^{-2 \omega^{4} t+2 \Pi(t) \omega^{2}}\left|\hat{u}_{0}(\omega)\right|^{2} d \omega+g^{\prime}(t),
$$

or in terms of $\xi$,

$$
\begin{aligned}
P(t) t^{-5 / 4} \int \xi^{4} e^{-2 \xi^{4}+2 \Sigma(t) \xi^{2}}\left|\hat{u}_{0}\left(\xi t^{-1 / 4}\right)\right|^{2} d \xi & \\
& =t^{-7 / 4} \int \xi^{6} e^{-2 \xi^{4}+2 \Sigma(t) \xi^{2}}\left|\hat{u}_{0}\left(\xi t^{-1 / 4}\right)\right|^{2} d \xi+g^{\prime}(t)
\end{aligned}
$$

Lemma 4.3 allows us to replace $\left|\hat{u}_{0}\left(\xi t^{-1 / 4}\right)\right|^{2}$ by $\hat{u}_{0}^{2}(0)$, so that

$$
t^{-7 / 4} \int \xi^{6} e^{-2 \xi^{4}+2 \Sigma(t) \xi^{2}}\left|\hat{u}_{0}\left(\xi t^{-1 / 4}\right)\right|^{2} d \xi+g^{\prime}(t) \sim t^{-7 / 4} u_{0}^{2}(0) F_{2}(\Sigma(t))+\frac{1}{2} g^{\prime}(t) .
$$

Now

$$
\begin{aligned}
t^{-7 / 4} u_{0}^{2}(0) F_{2}(\Sigma(t)) & \sim \frac{1}{4} t^{-7 / 4} \Sigma^{2} u_{0}^{2}(0) F_{0}(\Sigma) \\
& \sim \frac{1}{4} t^{-7 / 4} \Sigma^{2} h(t) \\
& \sim \frac{C}{4} t^{\gamma-1} \Sigma^{2}
\end{aligned}
$$


and since $\Sigma(t) \rightarrow \infty$, in the limit, $t^{-7 / 4} F_{2}(\Sigma)$ is larger than $g^{\prime}(t)$. Consequently, from (4.6),

$$
P(t) \sim t^{-1 / 2} \frac{F_{2}(\Sigma(t))}{F_{1}(\Sigma(t))} \sim \frac{1}{2} t^{-1 / 2} \Sigma(t)
$$

and by (4.5), this is equal to

$$
P(t) \sim \frac{1}{2} t^{-1 / 2} \sqrt{2(\gamma+3 / 4) \log t} \quad \text { as } t \rightarrow \infty .
$$

This concludes the proof of Theorem 4.2.

We still owe the reader the proof of Lemma 4.3.

Proof of Lemma 4.3. Define $\rho(\xi)=\sup \{|v(x)-v(0)|:-\xi<x<\xi\}$ for all $\xi \geq 0$. Since $v$ is continuous at the origin, $\rho(\xi) \rightarrow 0$ as $\xi \rightarrow 0$. Set $f_{n}(\xi, y)=\xi^{2 n+2} e^{-2 \xi^{4}+2 y \xi^{2}}$. Taking the difference of the two terms mentioned in the lemma,

$$
\begin{aligned}
\int f_{n}(\xi, y) \mid v(\xi / p) & -v(0) \mid d \xi \\
\leq & \rho(2 \sqrt{y} / p) \int_{|\xi| \leq 2 \sqrt{y}} f_{n}(\xi, y) d \xi+2\|v\|_{L^{\infty}(\mathbb{R})} \int_{|\xi|>2 \sqrt{y}} f_{n}(\xi, y) d \xi .
\end{aligned}
$$

The first integral on the right-hand side is bounded by $\rho(2 \sqrt{y} / p) F_{n}(y)$ and therefore is of the order $o\left(F_{n}(y)\right)$ as $y, p \rightarrow \infty$; the lemma is proved if we show the same for the second integral.

Denote the second integral by $I$. Under the change of variables $\xi=\eta y^{1 / 2}$, we can write

$$
I=y^{n+3 / 2} \int_{|\eta|>2} \eta^{2 n+2} e^{-2 y^{2}\left(\eta^{4}-\eta^{2}\right)} d \eta
$$

We estimate the exponential by

$$
\begin{aligned}
e^{-2 y^{2}\left(\eta^{4}-\eta^{2}\right)} & =e^{-2 y^{2}\left(\eta^{4} / 4-\eta^{2}\right)} \cdot e^{-\frac{3}{2} \eta^{4} y^{2}} \\
& \leq e^{-\frac{3}{2} y^{2}} \cdot e^{-\frac{3}{2} y^{2}\left(\eta^{4}-1\right)}
\end{aligned}
$$

so that

$$
y^{n+3 / 2} \int_{|\eta|>2} \eta^{2 n+2} e^{-2 y^{2}\left(\eta^{4}-\eta^{2}\right)} d \eta \leq y^{n+3 / 2} e^{-\frac{3}{2} y^{2}} \int_{\mathbb{R}} \eta^{2 n+2} e^{-\frac{3}{2}\left(\eta^{4}-1\right)} d \eta,
$$

where we suppose in the last inequality that $y \geq 1$. As a result,

$$
I \leq C y^{n+3 / 2} e^{-\frac{3}{2} y^{2}}
$$

for some constant $C>0$, which is clearly smaller than $F_{n}(y)$ as $y \rightarrow \infty$. This concludes the proof of the lemma.

In the next section the following convergence result will come in handy.

Lemma 4.4. Let $u_{0}$ satisfy the conditions of Theorem 4.2. Then

$$
\int \omega^{2}\left|\hat{u}_{0}(\omega)-\hat{u}_{0}(0)\right|^{2} e^{-2 \omega^{4} t+2 \Pi(t) \omega^{2}} d \omega=o(g(t)) \quad \text { as } t \rightarrow \infty .
$$


This lemma could be interpreted as stating that as far as the long-term behavior is concerned, the difference between

$$
\hat{u}(\omega, t)=\hat{u}_{0}(\omega) e^{-\omega^{4} t+\Pi(t) \omega^{2}}
$$

and

$$
\underline{\hat{u}}(\omega, t)=\hat{u}_{0}(0) e^{-\omega^{4} t+\Pi(t) \omega^{2}}
$$

is of smaller order than the norm of the solution $u$ itself, which is equal to $g(t)$ by definition.

Proof. Writing the integral as

$$
t^{-3 / 4} \int \xi^{2}\left|\hat{u}_{0}\left(\xi t^{-1 / 4}\right)-\hat{u}_{0}(0)\right|^{2} e^{-2 \xi^{4}+2 \Sigma(t) \xi^{2}} d \xi
$$

the result follows immediately from Lemma 4.3 and (4.4).

5. Long-term behavior: The profile $\boldsymbol{u}$. The asymptotic formulae that were obtained in the previous section enable us to derive the spatial structure of the solution $u$ as $t \rightarrow \infty$. The main result of this section (Theorem 5.1 and Corollary 5.2) is that in the limit $t \rightarrow \infty$ the profile $u(x, t)$ converges to

$$
\psi(x, t) \stackrel{\text { def }}{=} \sqrt{2 C}[\pi(\gamma+3 / 4) \log t]^{-1 / 4} P(t)^{-1 / 4} g(t)^{1 / 2} e^{-y^{2} / 8(\gamma+3 / 4) \log t} \cos y,
$$

where $y \stackrel{\text { def }}{=} x P^{1 / 2} \sim \sqrt{A} x t^{-1 / 4}[\log t]^{1 / 4}$ and $A=(\gamma+3 / 4) / 2$ (recall that $C$ is given in Theorem 4.2 by $\left.g(t) \sim C t^{\gamma}\right)$. Here the convergence is uniform on bounded sets in $y$.

To establish this we introduce the change of variables

$$
u(x, t)=P(t)^{-1 / 4} g(t)^{1 / 2} v(y, t) .
$$

For the sake of clarity we omit the $t$-dependence of $P, \Pi, \Sigma$, and $g$. In Fourier space, $\hat{u}$ and $\hat{v}$ are related by

$$
\hat{v}(\omega, t)=P^{3 / 4} g^{-1 / 2} \hat{u}\left(\omega P^{1 / 2}, t\right),
$$

and by inserting (3.4),

$$
\hat{v}(\omega, t)=P^{3 / 4} g^{-1 / 2} \hat{u}_{0}\left(\omega P^{1 / 2}\right) e^{-\omega^{4} P^{2} t+P \Pi \omega^{2}} .
$$

The function $\hat{v}$ now satisfies the constraint

$$
\frac{1}{2} \int \omega^{2}|\hat{v}|^{2} d \omega=\frac{1}{2 g} \int\left(\omega P^{1 / 2}\right)^{2}\left|\hat{u}\left(\omega P^{1 / 2}\right)\right|^{2} d \omega P^{1 / 2}=1 .
$$

We claim that $\hat{v}$ converges to

$$
\hat{z}(\omega, t)=\alpha(t)\left\{e^{-\beta(t)\left[4(\omega-1)^{2}-1\right]}+e^{-\beta(t)\left[4(\omega+1)^{2}-1\right]}\right\},
$$

where $\beta(t)$ is the solution of the equation

$$
\frac{e^{2 \beta}}{\sqrt{\beta}}=2 \sqrt{\frac{2}{\pi}} \hat{u}_{0}^{-2}(0) P^{-3 / 2} g
$$


and $\alpha(t)$ is chosen such that $\frac{1}{2} \int \omega^{2}|\hat{z}|^{2}=1$ for all $t$. We denote by $\hat{z}_{-}$and $\hat{z}_{+}$the two parts of $\hat{z}$. We will need the asymptotic form of $\alpha$ and $\beta$, so we will derive them here. From inverting (5.3) we find that

$$
\beta(t) \sim \frac{1}{2}\left(\log g-\frac{3}{2} \log P\right) \sim \frac{1}{2}(\gamma+3 / 4) \log t \quad \text { as } t \rightarrow \infty,
$$

and by applying (4.1) to $\alpha$ we find

$$
\begin{aligned}
1 \sim 2 \cdot \frac{1}{2} \int \omega^{2}\left|\hat{z}_{-}\right|^{2} & =2 \alpha^{2}(t) \int \omega^{2} e^{-2 \beta(t)\left[4(\omega-1)^{2}-1\right]} d \omega \\
& \sim \alpha^{2}(t) \sqrt{2 \pi} \frac{e^{2 \beta(t)}}{\sqrt{16 \beta(t)}},
\end{aligned}
$$

so that

$$
\alpha(t) \sim \hat{u}_{0}(0) P^{3 / 4} g^{-1 / 2} \quad \text { as } t \rightarrow \infty .
$$

As mentioned before, since $u_{0}$ is real, $\hat{u}_{0}(0)=\int u_{0}$ is also real.

The spatial form of the asymptotic profile is recovered by transforming back the function $\hat{z}$,

$$
\begin{aligned}
\hat{z}(\omega, t) & =\alpha(t) e^{\beta(t)}\left\{e^{-4 \beta(t)(\omega-1)^{2}}+e^{-4 \beta(t)(\omega+1)^{2}}\right\} \\
& =\alpha(t) e^{\beta(t)}(\delta(\omega-1)+\delta(\omega+1)) * e^{-4 \beta(t) \omega^{2}},
\end{aligned}
$$

so that

$$
z(y, t)=\alpha(t) e^{\beta(t)} \frac{1}{\sqrt{2 \beta(t)}} e^{-y^{2} / 16 \beta(t)} \cos y .
$$

Using the asymptotic formulae of the previous sections, we can derive a representation

$$
e^{-y^{2} / 16 \beta(t)} \cos y \sim e^{-y^{2} / 8(\gamma+3 / 4) \log t} \cos y \quad \text { for all } y \quad \text { as } t \rightarrow \infty,
$$

and using (5.3) and (5.5),

$$
z(y, t) \sim \sqrt{2}[\pi(\gamma+3 / 4) \log t]^{-1 / 4} e^{-y^{2} / 8(\gamma+3 / 4) \log t} \cos y \quad \text { for all } y \quad \text { as } t \rightarrow \infty .
$$

TheOREM 5.1. Let $u_{0}$ and $g$ satisfy the conditions of Theorem 4.2. Then

$$
\int \omega^{2}|\hat{v}(\omega, t)-\hat{z}(\omega, t)|^{2} d \omega \rightarrow 0 \quad \text { as } t \rightarrow \infty .
$$

Possibly more interesting is convergence in the uniform norm, as shown in the corollary.

COROllary 5.2. Under the conditions of the previous theorem,

$$
\|v(\cdot, t)-z(\cdot, t)\|_{L^{\infty}(\mathbb{R})} \rightarrow 0 \quad \text { as } t \rightarrow \infty .
$$

Proof of Theorem 5.1. Consider

$$
\int \omega^{2}|\hat{v}-\hat{z}|^{2}=\int \omega^{2}|\hat{v}|^{2}+\int \omega^{2}|\hat{z}|^{2}-2 \int \omega^{2} \hat{v} \bar{z} .
$$


Since the first two terms on the right-hand side are equal to 2 by construction, it is sufficient to show that

$$
\int \omega^{2} \hat{v} \bar{z} \rightarrow 2 \quad \text { as } t \rightarrow \infty
$$

Note also that if we define, in keeping with the remark after Lemma 4.4,

$$
\underline{\hat{v}}(\omega, t)=P^{3 / 4} g^{-1 / 2} \hat{u}_{0}(0) e^{-\omega^{4} P^{2} t+P \Pi \omega^{2}},
$$

then

$$
\begin{aligned}
\int \omega^{2}|(\hat{v}-\underline{\hat{v}}) \hat{z}| & \leq\left(\int \omega^{2}|\hat{v}-\underline{\hat{v}}|^{2}\right)^{1 / 2} \\
& =\left(\int \omega^{2}|\hat{u}-\underline{\hat{u}}|^{2}\right)^{1 / 2} \rightarrow 0 \quad \text { as } t \rightarrow \infty
\end{aligned}
$$

by Lemma 4.4. Therefore, we can replace $\hat{v}$ by $\underline{\hat{v}}$ in the integral in (5.6). We do so but immediately drop the underline again.

For a constant $R>0$ that we will choose later, we split the integral into different parts,

$$
\begin{aligned}
\int \omega^{2} \hat{v} \bar{z} & =\int_{-R}^{R} \omega^{2} \hat{v} \overline{\hat{z}}_{-}+\int_{-R}^{R} \omega^{2} \hat{v} \overline{\hat{z}}_{+}+\int_{|\omega|>R} \omega^{2} \hat{v} \overline{\hat{z}}_{-}+\int_{|\omega|>R} \omega^{2} \hat{v} \overline{\hat{z}}_{+} \\
& =I_{-}+I_{+}+J_{-}+J_{+} .
\end{aligned}
$$

First we investigate $J_{ \pm}$. Using the notation

$$
p(\omega)=4(\omega-1)^{2}-1+\omega^{4}-2 \omega^{2}
$$

and

$$
r(\omega, t)=-\omega^{4}+2 \omega^{2}+\frac{P^{2} t}{\beta(t)} \omega^{4}-\frac{P \Pi}{\beta(t)} \omega^{2},
$$

the integral $J_{-}$can be written as

$$
J_{-}=\hat{u}_{0}(0) P^{3 / 4} g^{-1 / 2} \alpha(t) \int_{|\omega|>R} \omega^{2} e^{-\beta(t)[p(\omega)+r(\omega, t)]} d \omega .
$$

From Theorem 4.2 and (5.4) we deduce that

$$
\frac{P^{2} t}{\beta(t)} \sim \frac{\frac{1}{2}(\gamma+3 / 4) \log t}{\frac{1}{2}(\gamma+3 / 4) \log t}=1
$$

and

$$
\frac{P \Pi}{\beta(t)} \sim \frac{(\gamma+3 / 4) \log t}{\frac{1}{2}(\gamma+3 / 4) \log t}=2,
$$

which together imply that (a) $r(\omega, t)$ tends to zero uniformly on compact sets as $t \rightarrow \infty$, and (b) $r(\omega, t) \geq-1 / 2 \omega^{4}-\omega^{2}$ for all $\omega \in \mathbb{R}$ if $t$ is large. We can now choose 
$R>0$ and $T>0$ large enough to have $p(\omega)+r(\omega, t) \geq 2 \omega^{2}$ for all $t \geq T$ and $|\omega| \geq R$. Then

$$
\left|J_{-}\right| \leq \hat{u}_{0}(0) P^{3 / 4} g^{-1 / 2} \alpha(t) \int_{|\omega|>R} \omega^{2} e^{-2 \beta \omega^{2}} d \omega .
$$

If $T$ is large enough, then

$$
\int_{|\omega|>R} \omega^{2} e^{-2 \beta \omega^{2}} d \omega \leq \int_{|\omega|>R} e^{-\beta \omega^{2}} d \omega=\frac{2}{\sqrt{\beta}} \operatorname{erfc}(R \sqrt{\beta}),
$$

and this last term tends to zero as

$$
\frac{2}{\sqrt{\beta}} \frac{1}{2 R \sqrt{\pi \beta}} e^{-\beta R^{2}} \sim \frac{2}{(\gamma+3 / 4) R \sqrt{\pi} \log t} t^{-\frac{1}{2}(\gamma+3 / 4) R^{2}} .
$$

Consequently, if $R$ is large enough, this term decreases fast enough to cancel a possible increase by $g^{-1 / 2}$; since in addition both $P$ and $\alpha$ tend to zero, the integral $J_{-}$tends to zero. The integral $J_{+}$is handled similarly.

Applying the same ideas to $I_{ \pm}$we write

$$
I_{-}=\hat{u}_{0}(0) P^{3 / 4} g^{-1 / 2} \alpha(t) \int_{-R}^{R} \omega^{2} e^{-\beta(t)[p(\omega)+r(\omega, t)]} d \omega
$$

and since $r \rightarrow 0$ uniformly on $[-R, R]$ as $t \rightarrow \infty$, we have

$$
I_{-}=(1+o(1)) \hat{u}_{0}(0) P^{3 / 4} g^{-1 / 2} \alpha(t) \int_{-R}^{R} \omega^{2} e^{-\beta(t) p(\omega)} d \omega .
$$

By (4.1),

$$
I_{-} \sim \hat{u}_{0}(0) P^{3 / 4} g^{-1 / 2} \alpha(t) \sqrt{2 \pi} \frac{e^{-2 \beta(t)}}{\sqrt{16 \beta(t)}},
$$

and by inserting (5.3) and (5.5),

$$
I_{-} \sim \hat{u}_{0}(0) P^{3 / 4} g^{-1 / 2} \cdot \hat{u}_{0}(0) P^{3 / 4} g^{-1 / 2} \cdot \hat{u}_{0}^{-2}(0) P^{-3 / 2} g=1 .
$$

The same argument applies to $I_{+}$, by which Theorem 5.1 is proved.

The corollary follows simply from the dominated convergence theorem.

Proof of Corollary 5.2. From Theorem 5.1 it follows that $\hat{v}-\hat{z} \rightarrow 0$ almost everywhere (a.e.) on $(-1 / 2,1 / 2)$. Estimating the difference in the $L^{2}$-norm,

$$
\int|\hat{v}(\omega, t)-\hat{z}(\omega, t)|^{2} d \omega \leq \int_{-1 / 2}^{1 / 2}|\hat{v}-\hat{z}|^{2}+4 \int_{|\omega|>1 / 2} \omega^{2}|\hat{v}-\hat{z}|^{2}
$$

we find that the first term converges to zero since $\hat{v}-\hat{z}$ is bounded on $(-1 / 2,1 / 2)$ by the continuity of $\hat{u}_{0}$ at $\omega=0$. The second term converges to zero by Theorem 5.1.

Consequently $v-z$ converges to zero in the $H^{1}$-norm, and the uniform convergence then follows from a well-known Sobolev embedding theorem. 
6. Long-term behavior for the half-space model. In the case of a foundation consisting of a viscous half-space, the results are very similar. Recall the problem, Find $(u, P)$ such that

$$
H\left(u_{x t}\right)+u_{x x x x}+P u_{x x}=0, \quad x \in \mathbb{R}, \quad t>0,
$$

with initial condition

$$
u(x, 0)=u_{0}(x), \quad x \in \mathbb{R},
$$

such that $u$ satisfies the constraint

$$
\frac{1}{2} \int_{\mathbb{R}} u_{x}^{2} d x=g(t), \quad t>0 .
$$

By virtue of the Fourier representation of the Hilbert transform,

$$
(H(u))^{r}(\omega)=-i \operatorname{sgn}(\omega) \hat{u}(\omega),
$$

the first term in (6.1) has a transform $|\omega| \hat{u}_{t}(\omega)$, so that we obtain an equation very similar to (3.3),

$$
|\omega| \hat{u}_{t}+\left(\omega^{4}-P(t) \omega^{2}\right) \hat{u}=0 .
$$

We find the solution

$$
\hat{u}(\omega, t)=\hat{u}_{0}(\omega) e^{-|\omega|^{3} t+\Pi(t)|\omega|},
$$

and $\Pi$ is determined by the integral equation

$$
\frac{1}{2} \int \omega^{2} e^{-2|\omega|^{3} t+2 \Pi(t)|\omega|}\left|\hat{u}_{0}(\omega)\right|^{2} d \omega=g(t) .
$$

Because of the similarity with the Winkler case, the results are practically the same, and there is no difference in the techniques used in the proofs. Consequently we will just state the results without proof.

Theorem 3.1 holds unchanged for the half-space case. In Theorem 3.2 the formulas become

$$
P(t)=\left[g^{\prime}(t)+\int|\omega|^{5}|\hat{u}|^{2}\right]\left(\int|\omega|^{3}|\hat{u}|^{2}\right)^{-1}
$$

and

$$
P^{\prime}(t)=\left[g^{\prime \prime}(t)-4 \int \omega^{2}\left|\hat{u}_{t}\right|^{2}\right]\left(\int|\omega|^{3}|\hat{u}|^{2}\right)^{-1} .
$$

The long-term behavior of $P$ is given by

$$
P \sim 2^{-4 / 3} t^{-2 / 3}[(1+\gamma) \log t]^{2 / 3},
$$

and if $v$ is given by

$$
u(x, t)=P^{-1 / 4} g^{1 / 2} v\left(x P^{1 / 2}, t\right),
$$

then $v(y, t)$ converges to the limit profile

$$
2^{5 / 4}[3 \pi(1+\gamma) \log t]^{-1 / 4} e^{-y^{2} / 3(1+\gamma) \log t} \cos y .
$$


7. Nonexistence of self-similar solutions. As mentioned above, (3.1) has a scaling invariance

$$
t \rightarrow \lambda t, \quad x \rightarrow \lambda^{1 / 4} x, \quad P \rightarrow \lambda^{-1 / 2} P .
$$

If we add the scaling $u \rightarrow \lambda^{1 / 8} u$, then this scaling also leaves the integral (3.2) invariant. A natural question in this situation is whether there exist any self-similar solutions with this scaling, i.e., solutions of the type

$$
u(x, t)=t^{1 / 8} f\left(x t^{-1 / 4}\right), \quad P=Q t^{-1 / 2} .
$$

The long-term convergence result of the previous sections shows that this cannot be the case - for how can a self-similar solution have a non-self-similar asymptotic behavior? - but we wish to prove this nonexistence in a more direct way.

A solution of the type (7.1), with constant $g$, would have a profile $f$ that satisfies the equation

$$
f^{\prime \prime \prime \prime}+Q f^{\prime \prime}+\frac{1}{8} f-\frac{1}{4} \eta f^{\prime}=0, \quad-\infty<\eta<\infty,
$$

and in addition the integral constraint

$$
\frac{1}{2} \int f^{\prime}(\eta)^{2} d \eta=g
$$

Applying the Fourier transform to this equation we find

$$
\left(\omega^{4}-Q \omega^{2}+\frac{3}{8}\right) \hat{f}+\frac{1}{4} \omega \hat{f}^{\prime}=0,
$$

where the last term is obtained by using $\hat{\eta}=i \sqrt{2 \pi} \delta^{\prime}$,

$$
\begin{aligned}
\left(\eta f^{\prime}\right)^{\prime}(\omega) & =\frac{1}{\sqrt{2 \pi}}(\hat{\eta} * i \omega \hat{f})(\omega) \\
& =-\left(\delta^{\prime} * \omega \hat{f}\right)(\omega) \\
& =-\left(\delta *(\omega \hat{f})^{\prime}\right)(\omega) \\
& =-\omega \hat{f}^{\prime}(\omega)-\hat{f}(\omega) .
\end{aligned}
$$

If $\hat{f}$ is a nonzero solution of (7.4), then

$$
\frac{\hat{f}^{\prime}}{\hat{f}}=-\frac{3}{2} \omega^{-1}-4 \omega^{3}+4 Q \omega .
$$

Near $\omega=0$ the term $-3 / 2 \omega^{-1}$ dominates, so that

$$
\hat{f}(\omega) \sim|\omega|^{-3 / 2} \quad \text { as } \omega \rightarrow 0 .
$$

Consequently,

$$
\int f^{\prime}(\eta)^{2} d \eta=\int \omega^{2}|\hat{f}(\omega)|^{2} d \omega=\infty
$$

implying that condition (7.3) cannot be satisfied. 
We can extend this remark to self-similar solutions with nonconstant constraint functions

$$
g(t)=c t^{\gamma}
$$

Solutions are of the form

$$
u(x, t) \sim t^{1 / 8+\gamma / 2} f\left(x t^{-1 / 4}\right), \quad P \sim Q t^{-1 / 2},
$$

and the profile $f$ satisfies

$$
\left[\omega^{4}-Q \omega^{2}+\frac{1}{2}\left(\gamma+\frac{3}{4}\right)\right] \hat{f}+\frac{1}{4} \omega \hat{f}^{\prime}=0 .
$$

By the same reasoning,

$$
\hat{f}(\omega) \sim|\omega|^{-3 / 2-2 \gamma} \quad \text { as } \omega \rightarrow 0
$$

and here we find that the parameter range of Theorems 4.2 and 5.1, $\gamma>-3 / 4$, exactly corresponds to the range in which the candidate self-similar solution has a singularity at the origin (in Fourier space).

We interpret this fact in the following way. Equation (3.1) conserves mass, i.e.,

$$
\int u(x, t) d x=\int u_{0}(x) d x \text { for all } t \geq 0 .
$$

The solutions we consider in this paper have finite mass, since we assume that $\hat{u}$ is continuous at $\omega=0$. We expect the conservation of mass to be preserved in the characterization of the long-term behavior; therefore, any self-similar solution that is a candidate for the long-term behavior should have finite and constant mass. This corresponds exactly to the condition that $\hat{f}$ should have no singularity at the origin.

To conclude, in the range $\gamma>-3 / 4$ there exist self-similar solutions of (3.1); but for $\gamma \geq 0$ they have an infinite integral $\int f^{\prime 2}$, and therefore do not satisfy the constraint condition. If $-3 / 4<\gamma<0$, then the integral $\int f^{\prime 2}$ has a finite value, but the solution has infinite mass and is therefore not a candidate for the long-term behavior.

It is clear, both from the argument above and from the proof of Theorem 4.2, that the other parameter range, $\gamma \leq-3 / 4$, is completely different. We conjecture that here the long-term behavior of solutions is self-similar, and we expect to return to this question in a future publication.

8. Numerical approximation. For the numerically calculated solutions in this paper we restrict the problem to a finite domain $(-L, L)$, with $L$ suitably large, and impose the boundary conditions of a simply supported beam $\left(u( \pm L)=u_{x x}( \pm L)=0\right)$. An equivalent variational formulation of the Winkler model equation (3.1) follows by multiplying the equation by a test function $v$ with $v( \pm L)=0$ and integrating:

$$
\int_{-L}^{L} u_{t} v d x+\int_{-L}^{L} u_{x x} v_{x x} d x-P \int_{-L}^{L} u_{x} v_{x} d x=0
$$

We now determine an approximation to $u(x, t)$ by using the finite-element method to give a semidiscretization of (8.1) [21]. To do this we approximate $u(x, t)$ by the 
function $U_{h}(x, t)=\sum U_{i}(t) \phi_{i}(x)+\sum U_{x i}(t) \psi_{i}(x)$. Here $\phi_{i}$ and $\psi_{i}$ are piecewise cubic functions defined on a uniform mesh of spacing $h \stackrel{\text { def }}{=} 2 L / N$ so that

$$
\begin{array}{r}
\phi_{i}(-L+j h)=\psi_{i}^{\prime}(-L+j h)=\delta_{i j} \quad \text { and } \quad \psi_{i}(-L+j h)=\phi^{\prime}(-L+j h)=0 \\
\text { for } i, j=0, \ldots, N .
\end{array}
$$

The space $S_{h}$ is the span of the functions $\phi_{i}(i=1, \ldots, N-1)$ and $\psi_{i}(i=0, \ldots, N)$ (such that the imposed boundary condition $u=0$ is incorporated into the solution space). We set $U \in \mathbb{R}^{2 N}$ equal to $U=U(t)=\left(U_{1}, \ldots, U_{N-1}, U_{x 0}, \ldots, U_{x N}\right)$. Now we require that $U_{h}$ should satisfy (8.1) for all functions $V \in S_{h}$. Setting $V=\phi_{i}$ or $V=\psi_{i}$, this leads to the following system of ordinary differential equations for $U$ and $P$ :

$$
A U_{t}+B U-P C U=0,
$$

where the $2 N \times 2 N$ matrices $A, B$, and $C$ are given by

$$
\begin{aligned}
A_{i j}=\int \phi_{i} \phi_{j}, & 1 \leq i, j \leq N-1, \\
B_{i j}=\int \phi_{i}^{\prime \prime} \phi_{j}^{\prime \prime}, & 1 \leq i, j \leq N-1, \\
C_{i j}=\int \phi_{i}^{\prime} \phi_{j}^{\prime}, & 1 \leq i, j \leq N-1,
\end{aligned}
$$

with similar entries for other ranges of $i$ and $j$.

The axial load $P(t)$ is determined as part of the solution and the necessary and sufficient condition comes from the integral constraint (3.2), which reads in discretized form

$$
\frac{1}{2} U^{T} C U=g(t)
$$

The system (8.2)-(8.3) is then an index-2 differential-algebraic equation (DAE). Differentiating (8.3) with respect to time, we find

$$
U^{T} C U_{t}=g^{\prime}(t)
$$

We solved (8.2) and (8.4) using DDASSL, a backward-difference form DAE solver [17]. We choose to replace the constraint (8.3) by (8.4) since the latter provides a DAE system of index 1 , which DDASSL is designed to handle. It is verified after calculation that the deviation from (8.3) due to accumulation of numerical error is acceptably small (relative error less than 0.1).

8.1. Constant end-shortening. Figure 8.1 shows an example of the evolution of $u$ and $P$ from a localized initial datum. With random but localized initial data the same trends can be recognized (Figure 8.2). The form of the asymptotic behavior can be better appreciated in scaled coordinates. Inspired by the transformation (5.2) we plot $P^{1 / 4} u(x, t)$ against $x P^{1 / 2}$ (Figure 8.3). In Figure 8.4 we compare the numerically calculated solution against the profile $\psi$ given by (5.1). There is a strong agreement between the two, in both amplitude and phase, thus supporting both the asymptotic and the numerical calculations. 

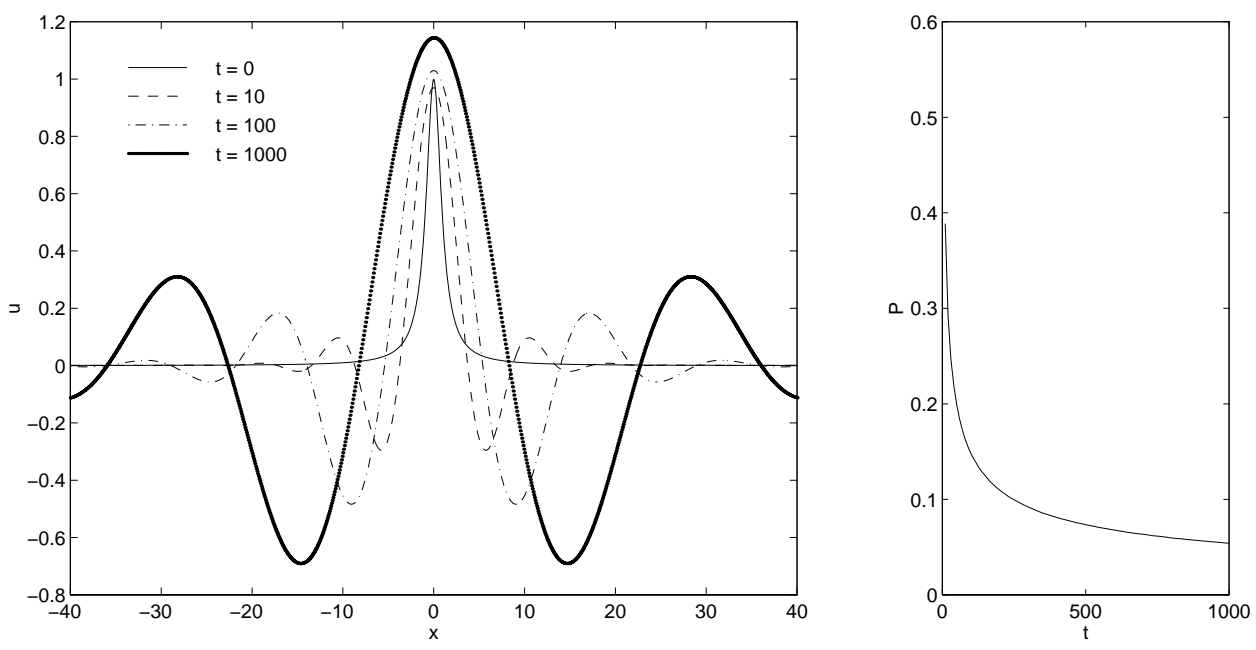

FIG. 8.1. Evolution of the solution profile $u$ and the layer-parallel load P. The initial datum $u_{0}(x)$ is equal to $1 /\left(1+x^{2}\right)$ and the end-shortening is constant. The spatial domain was $[-100,100]$, divided into 256 elements of equal size, and the time integration was done with a relative error tolerance of $10^{-4}$.
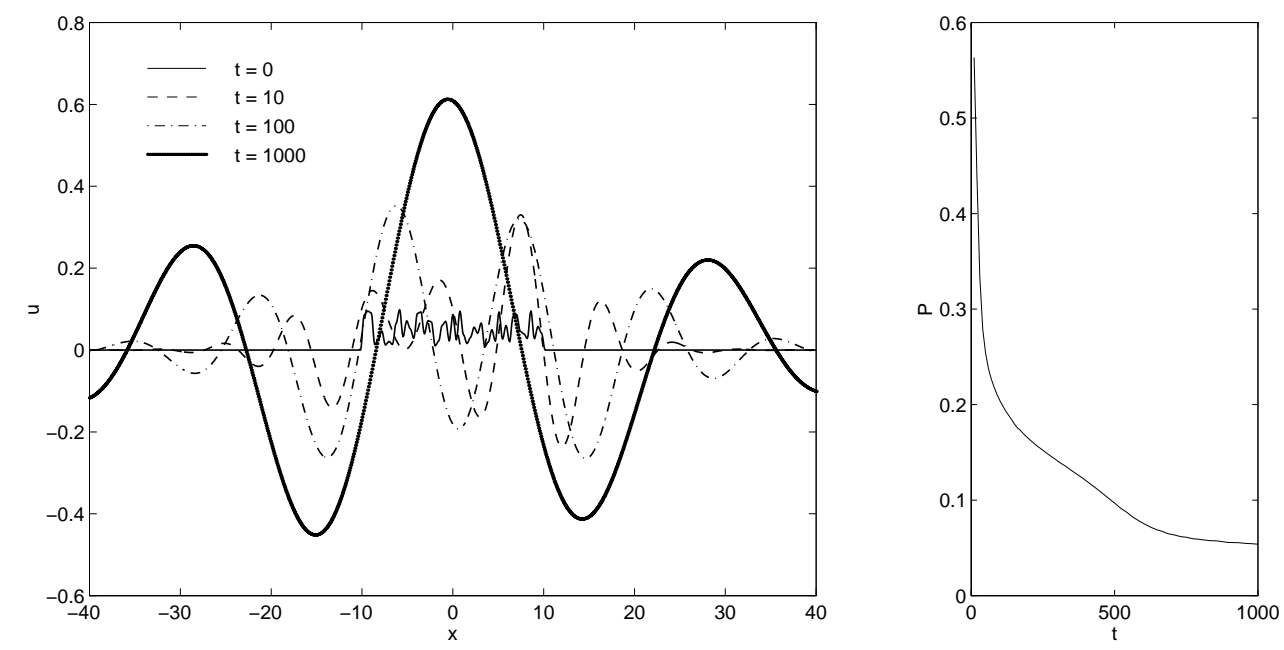

FIG. 8.2. Evolution from random initial data: $U_{i}(0)$ (see text) takes random values on the interval $[-10,10]$, with maximum 0.1. Discretization data the same as Figure 8.1.

8.2. Advancing end-shortening. Figure 8.5 shows the evolution from the same initial data as Figure 8.1, but with a linearly advancing end-shortening condition $g(t)=(1+t) \pi / 4$ (note that $\int u_{0 x}^{2} d x=\pi / 4$ ). As predicted by the appearance of the factor $g(t)^{1 / 2}$ in (5.1), there is a stronger increase in amplitude than in the constant end-shortening case.

8.3. Consequences of the finite-domain approximation. In the calculations described above it is obviously wise to choose a spatial domain that is large enough for boundary effects to be negligible. Since solutions "spread out" in time, both in the original and in the scaled variables, it is to be expected that after finite 


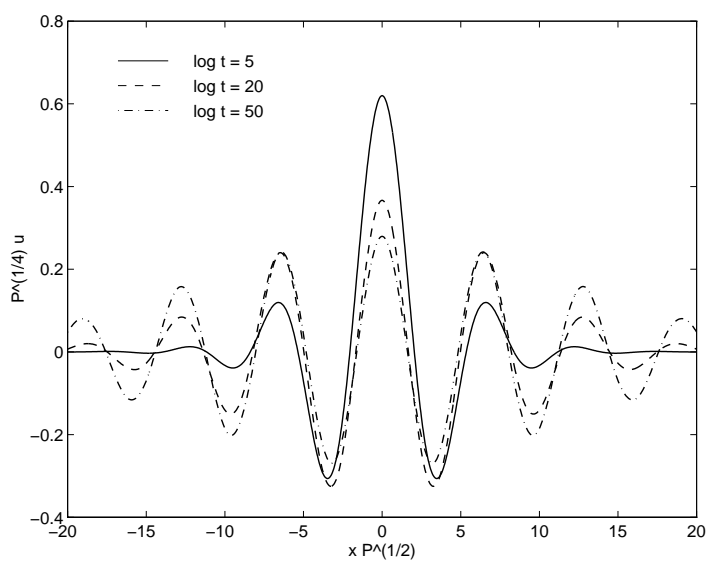

FIG. 8.3. Same evolution as in Figure 8.1, but plotted in scaled coordinates.

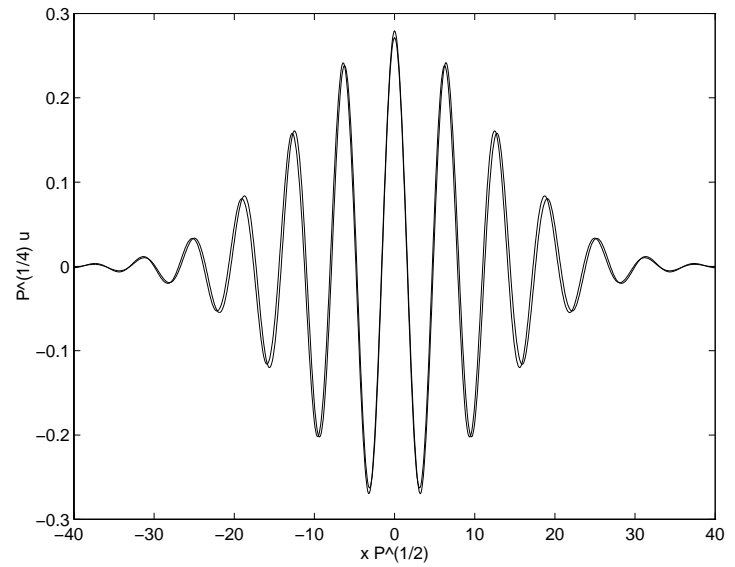

FIG. 8.4. Comparison between the calculated solution and the asymptotic solution $\psi$ at time $\log t=50$.

time the effect of the boundary should become noticeable. This is indeed so, but the way in which the boundary influences the solution is strongly dependent on the choice of variables.

In the original spatial variables, the finite domain leads to a delocalization of the solution: in the course of the "spreading" evolution the boundaries create an instability that causes the solution to jump away from the localized packet of oscillations. After this jump it quickly converges to a stationary solution that has no sign changes and is quite close to a sinusoid half-wave.

In the scaled spatial variables the influence takes a different form. (Note that the two are not equivalent: fixed boundaries in the scaled variable $y=x P^{1 / 2}$ correspond to diverging boundaries in terms of $x$.) Again the boundaries create an instability, but instead of leading to a stationary solution, the evolution converges to what appears to be a low-dimensional invariant manifold. 

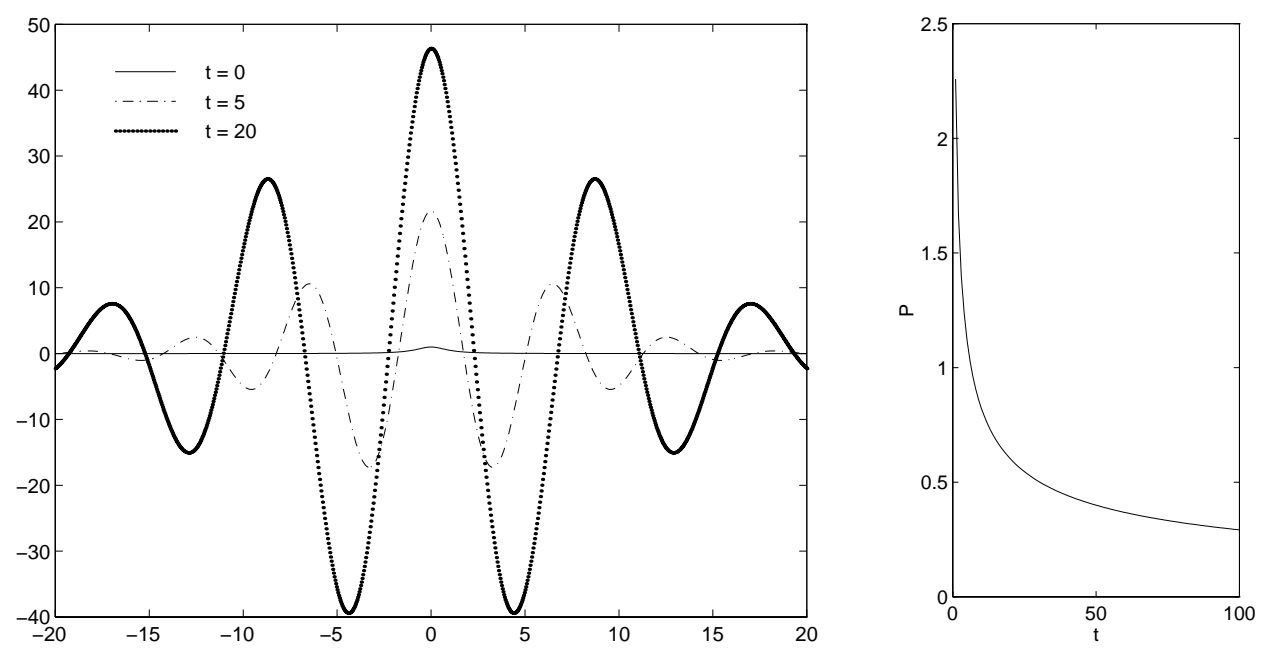

FIG. 8.5. Same evolution as Figure 8.1, but with advancing end-shortening constraint $g(t)=$ $(1+t) \pi / 4$.

Finally, we were surprised to see that the instability appears at a very early stage, when the values of the solution near the boundary are very small (typically $\sim 10^{-30}$ ).

9. Conclusion. The model presented here is very simple, and although in many aspects it compares favorably to established ideas in geology, the results presented here leave obvious room for extension, both in analysis and in modeling.

The long-term behavior described in this paper is expected to apply not only to the viscous foundation of this model, but also to foundations that are viscoelastic, and also, in some cases, to geometrically nonlinear models (as explained in the introduction). However, a long-term result begs the question of the short-term character of the model, and we hope to explore this question in the future. It should be noted that here the material properties are not irrelevant, and the difference between a viscoelastic and a viscous foundation should be visible.

A different extension, one that is hinted at in recent geological literature [6], would be to consider not only the foundation but also the layer to be viscoelastic. This would change the large-time behavior in a fundamental way, since the strain energy contained in the deformed layer can then dissipate viscously in both the foundation and the layer. The ratio between the two viscosities is expected to be of prime importance in such a model.

Again a different, but equally interesting extension would consist in relaxing the small-deflection hypothesis (1.2), resulting in a geometrically nonlinear model for the layer. Numerical results by Whiting and Hunt [23] show interesting behavior, especially for a relatively rapid increase in end-shortening. Here again, elastic properties could be significant.

Finally, the folding behavior of multilayers is fundamentally different from single layers. Interestingly, interplay between elastic and viscous effects seems to be important in this case, too. We are currently investigating this question.

Acknowledgments. This paper would not have appeared without the stimulating support and ideas of Prof. G. W. Hunt, who brought this problem to us and provided many insights into the underlying geological modeling. The authors also wish 
to express their gratitude to Prof. J. Mallet-Paret for a suggestion that simplified the proofs considerably.

\section{REFERENCES}

[1] G. I. Barenblatt, Similarity, Self-Similarity, and Intermediate Asymptotics, Consultants Bureau, New York, 1979.

[2] J. Bebernes AND S. BRICher, Final time blowup profiles for semilinear parabolic equations via center manifold theory, SIAM J. Math. Anal., 23 (1992), pp. 852-869.

[3] М. A. Віот, Theory of folding of stratified viscoelastic media and its implications in tectonics and orogenesis, Geol. Soc. Amer. Bull., 72 (1961), pp. 1595-1620.

[4] M. A. Віот, Mechanics of Incremental Deformations, Wiley, New York, 1965.

[5] R. S. Carmichael, Handbook of Physical Properties of Rocks, CRC Press, Boca Raton, FL, 1981-1984.

[6] H.-B. Mühlhaus, H. Sakaguchi, and B. E. Hobbs, Evolution of three-dimensional folds for a non-Newtonian plate in a viscous medium, Proc. Roy. Soc. Lond. Ser. A, 454 (1998), pp. 3121-3143.

[7] J. W. DoLD, Analysis of the early stage of thermal runaway, Quart. J. Mech. Appl. Math., 38 (1985), pp. 361-387.

[8] B. E. Hobbs, W. D. Means, and P. F. Williams, An Outline of Structural Geology, Wiley, New York, 1976.

[9] G. W. Hunt, G. J. Lord, And A. R. Champneys, Homoclinic and heteroclinic orbits underlying the post-buckling of axially-compressed cylindrical shells, Comput. Methods Appl. Mech. Engrg., 170 (1999), pp. 239-251.

[10] G. W. Hunt, H.-B. Mühlhaus, And A. I. M. Whiting, Evolution of localized folding for a thin elastic layer in a softening visco-elastic medium, Pure Appl. Geophys., 146 (1996), pp. 229-252.

[11] A. M. Johnson and R. C. Fletcher, Folding of Viscous Layers, Columbia University Press, New York, 1994.

[12] G. J. Lord, A. R. Champneys, and G. W. Hunt, Computation of localized post buckling in long axially-compressed cylindrical shells, in Localization and Solitary Waves in Solid Mechanics, A. R. Champneys, G. W. Hunt, and J. M. T. Thompson, eds., Special Issue of Philos. Trans. Roy. Soc. London Ser. A, 355 (1997), pp. 2137-2150.

[13] G. J. Lord, A. R. Champneys, and G. W. Hunt, Computation of homoclinic orbits in partial differential equations: An application to cylindrical shell buckling, SIAM J. Sci. Comput., 21 (1999), pp. 591-619.

[14] H. B. MÜHLHAUS, Evolution of elastic folds in plane strain, in Modern Approaches to Plasticity, D. Kolymbas, ed., Elsevier Science Publishers B.V., Amsterdam, 1993, pp. 737-765.

[15] H.-B. MüHlhaus, B. E. HobBs, AND A. ORD, The role of axial constraints on the evolution of folds in single layers, in Computer Methods and Advances in Geomechanics, Vol. 1, H. J. Siriwardane and M. M. Zaman, eds., A. A. Balkema, Rotterdam, the Netherlands, 1994, pp. 223-231.

[16] F. W. J. Olver, Asymptotics and Special Functions, Academic Press, New York, 1974.

[17] L. R. Petzold, A Description of DDASSL: A Differential/Algebraic System Solver, Tech. Rep. SAND82-8637, Sandia National Laboratories, Livermore, CA, 1982.

[18] N. J. Price and J. W. Cosgrove, Analysis of Geological Structures, Cambridge University Press, Cambridge, UK, 1990.

[19] J. G. RAmSAY, A geologist's approach to rock deformation, in Inaugural Lectures-Imperial College of Science, Technology and Medicine, London, 1967, pp. 131-143.

[20] J. M. T. Thompson and G. W. Hunt, A General Theory of Elastic Stability, Wiley, London, 1973.

[21] R. Wait And A. R. Mitchell, Finite Element Analysis and Applications, Wiley, New York, 1985.

[22] A. I. M. Whiting, Localized Buckling of an Elastic Strut in a Visco-Elastic Medium, Ph.D. thesis, Imperial College of Science, Technology and Medicine, London, 1996.

[23] A. I. M. Whiting AND G. W. Hunt, Evolution of nonperiodic forms in geological folds, J. Math. Geology, 29 (1997), pp. 705-723. 